\title{
Laboratory Test Sponsor Defined Identifier
}

National Cancer Institute

\section{Source}

National Cancer Institute. Laboratory Test Sponsor Defined Identifier. NCI Thesaurus.

Code C83316.

One or more sponsor defined characters used to name a laboratory test. 